\title{
Molecular Dynamics Simulations of the Protonated G4 PAMAM Dendrimer in an Ionic Liquid System
}

\author{
Juan J. Freire, ${ }^{* \dagger}$ Amirhossein Ahmadi, ${ }^{\dagger}$ and Carl McBride ${ }^{\dagger, \dagger}$ \\ ${ }^{\dagger}$ Departamento de Ciencias y Técnicas Fisicoquímicas, Facultad de Ciencias, Universidad Nacional de Educación a Distancia \\ (UNED), Paseo Senda del Rey 9, 28040 Madrid, Spain \\ ${ }^{\ddagger}$ Instituto de Química Física Rocasolano (CSIC), Serrano 119, 28006 Madrid, Spain
}

Supporting Information

ABSTRACT: Molecular dynamics simulations have been carried out for the ionic liquid system constituted by totally protonated PAMAM-EDA cations and Tf $2 \mathrm{~N}^{-}$ anions. The conformational characteristics of the PMAM dendrimer (particularly the density profile around the dendrimer center) are compared with those obtained for the same dendrimer in water. We also investigate other features, such as the location of anions relative to the dendrimer molecules, and the interpenetration of the dendrimer cations in the ionic liquid system. It is shown that a substantial amount of anions are present in the outer part of the dendrimer, screening repulsive contacts between different cation molecules and favoring ionic conductivity. Dendrimer cations in the ionic liquid exhibit a significant degree of overlap.

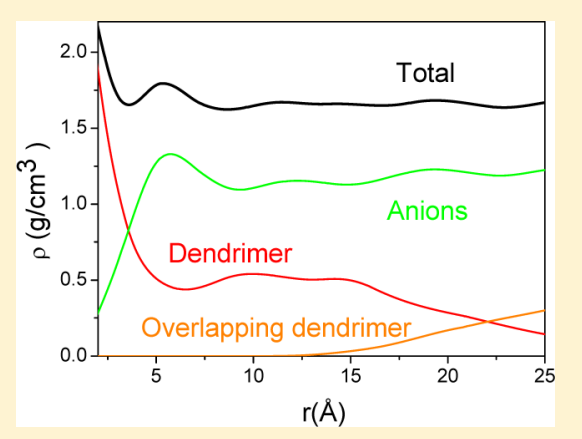

\section{INTRODUCTION}

Dendrimer molecules have enormous potential for a variety of applications because of their peculiar conformational properties with respect to other polymeric materials. ${ }^{1}$ The family of polyamidoamine dendrimers with a ethylenediamine core (PAMAM-EDA) has been the first to be commercially available. In these particular dendrimers, both primary and tertiary (or peripheral and inner) amine groups can be protonated in water solution and this protonation is strongly influenced by $\mathrm{pH}$ and other environmental conditions. All amine groups are expected to be fully protonated at low $\mathrm{pH}$ whereas they should remain neutral in high $\mathrm{pH}$ solutions. ${ }^{2}$

Ionic liquids (IL), defined as salts that melt at (or below) room temperature, have received a great deal of attention over the past few years due to their special properties, in particular their high conductivity and low vapor pressure, which makes them useful for many applications. ${ }^{3}$ A novel type of IL, composed of completely protonated G4 (i.e., including four generations) PAMAM-EDA dendrimer polycation together with bis(trifluoromethylsulfonyl)imide $\left(\mathrm{Tf} 2 \mathrm{~N}^{-}\right)$anions has been described in the literature. ${ }^{4}$ The ionic liquid behavior of this system has been confirmed by its high conductivity and also through NMR and IR spectroscopy. It is claimed that this system has an increased surface hydrophobic behavior due to the massive presence of the $\mathrm{Tf} 2 \mathrm{~N}^{-}$moieties. Moreover, it shows strong blue luminescence.

Given the great interest of this particular IL and the possibility of association of polycationic dendrimers in ionic liquids in a general context, we have performed molecular dynamics (MD) numerical simulations of the system. Numerical simulations have the advantage of predicting some properties that are not readily accessible from experiments, such as the detailed structure in the interior of a dendrimer molecule, including free-space and distribution of solvent molecules and counterions. We have explored how much the molecular structure of the dendrimer and the location of anions both in the interior and on the surface of the dendrimer differ from the usual structure in dendrimer solutions. Moreover, by considering a simulation system with two dendrimers, we have carried out an initial study of the interpenetration and mutual disposition of the polycations.

\section{MODELS AND METHODS}

Atomistic interactions can be described through the potential energy terms contained in force fields. The particular case of the G4 PAMAM-EDA molecule in water has been extensively studied considering different degrees of protonation. ${ }^{2}$ Early simulations $^{5}$ for this system yielded very different sizes depending on the protonation level. However, these results were in contradiction with experimental estimates obtained from small angle neutron scattering (SANS) measurements ${ }^{6}$ that show a small variation of the dendrimer size with $\mathrm{pH}$. This lack of significant influence of $\mathrm{pH}$ on the dendrimer size was also previously observed in some experiments with dendrimers with higher generation number. ${ }^{7} \mathrm{New}$ simulation results have achieved a better agreement with the experimental data for the particular $G=4$ case. $^{8}$ These new results were based on a modification of the general purpose, or universal, Dreiding force field, ${ }^{9}$ which explicitly considers the formation of some types of hydrogen bonds. Nevertheless, recent combined SANS

Received: March 14, 2013

Revised: November 14, 2013

Published: November 14, 2013 
and small-angle X-ray scattering (SAXS) data show a moderate increase in size due to protonation for other generation numbers. $^{10}$

We have investigated the possibility of implementing a similar specification of hydrogen bonds in the alternative, more specific, class II force field, PCFF. ${ }^{11}$ To verify if this description is adequate for the water solution system, we have performed some simulations for these systems, using the TIP3P molecular model parameters and partial charges for an explicit description of the water atoms. ${ }^{12}$ Results from these calculations are reported here. As with the most recent previous simulations, we have found a better reproduction of the neutron scattering data for $G=4$ for neutral and protonated PAMAM-EDA dendrimers when the hydrogen bonds are included. Our analysis of these systems includes properties and features not previously investigated, providing a broad description of the atomic distribution of masses around the dendrimer and also of the degree of penetration of the water molecules and their preferential association with the amine groups. The results for these properties are used as references in our subsequent investigation of the IL system.

In our study of the dendrimer IL system, we have considered a description of dendrimers based on the PCFF, both with and without hydrogen bonds. To describe $\mathrm{Tf}_{2} \mathrm{~N}^{-}$ions, we have used specific force-field parameters and partial charges that have been used in previous MD simulations ${ }^{13}$ for simpler IL systems, adapted to be consistent with the PCFF.

We prepare our simulations using the Materials Studio $(\mathrm{MS})^{14}$ software suite. We easily construct the initial configuration of subsequently higher generations for each dendrimer structure in vacuum using the building tools contained in the MS module "Visualizer". The "Visualizer" module also allows us to build the final protonated structures for the G4 dendrimers through the assignment of formal charge +1 for the $\mathrm{N}$-amine atoms and addition of new hydrogen atoms. The resulting configurations are subsequently minimized. Minimizations are performed with the "Discover" module included in the MS suite, and they are followed by short (20000 fs) MD simulations (also with module "Discover") to relax the structures. In these previous steps in a vacuum, we have employed a high dielectric constant to prevent a dendrimer contraction and allow for sufficiently open initial conformations. A careful choice of the initial configuration is critical, especially in the case of neutral dendrimers for which we have verified that initial compact structures are not sufficiently exposed to solvent interchange. For this particular case, we have prepared an open initial conformation by reversing the charge and hydrogen assignations in a protonated dendrimer that has been previously equilibrated to be sufficiently open. Employing the MS module "Amorphous Cell”, we construct relaxed simulation boxes with cubic periodic boundary conditions.

For the solution systems, we consider a single dendrimer (neutral or protonated) with 4000 water molecules. The system corresponding to a protonated dendrimer molecule in water also includes $126 \mathrm{Cl}^{-}$counterions. Charges are calculated according to the charge equilibration method. ${ }^{15}$ For the IL boxes, we include 2 dendrimers together with $252 \mathrm{Tf} 2 \mathrm{~N}^{-}$ anions that neutralize all the charged (primary and tertiary) amine groups present in the dendrimer cations. In the systems including protonated dendrimers (models $1-3$ ), the anions are initially located close to the amine groups. Also, we have prepared an IL system where the anions are initially placed at random locations (model 4). In Figure 1, we present snapshots of the initial configurations for models $1-3$ and 4 . It can be
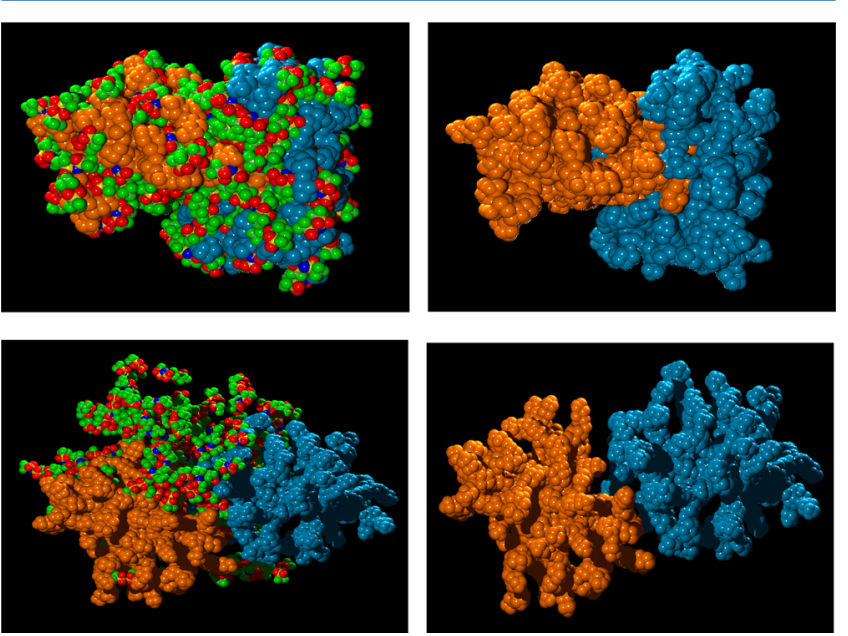

Figure 1. Snapshots of the initial IL systems: above, models 1-3 (anions close to the imine groups, with and without solvent); below, model 4 (anions located at random, with and without solvent). One dendrimer is pained blue and the other tan.

observed a significant degree of dendrimer overlapping in models 1-3 and a peripherical region of anions in model 4 . Furthermore, we have also considered a simulation box that only includes a single dendrimer molecule.

Although the module MS "Discover" can also perform MD simulations, we use the open source MD software DL-POLY ${ }^{16}$ for our more extensive MD runs. This software offers total flexibility when it comes to incorporating new force-field parameters. Moreover, we have verified that its use on Linux platforms is consistently more efficient from a computational point of view (the simulations being about a $2-3$ times faster when using the same number of parallel processors). The $D L$ $P O L Y$ input files are prepared from our previously generated MS "Discover" files. ${ }^{17}$

We carry out an NVT simulation to equilibrate the simulation box, followed by an NPT simulation to reach a stable value of the system density at room temperature (298 $\mathrm{K})$. We include as Supporting Information the density with respect to time in these initial equilibration runs. It can be observed that the curves show stable oscillations around fixed mean values in the final part of the trajectories. Typically, each one of the preliminary MD simulations consisted of at least $10^{6}$ simulations steps of 1 fs (i.e., 1 ns). We make use of the Berendsen thermostat and barostat (for NPT runs) and consider standard values of $r_{\mathrm{C}}=9.5 \AA$ for the intermolecular potential cutoff. Finally, we perform another more lengthy NVT simulation that generates a working trajectory for analysis (extending the total simulation time up to $20 \mathrm{~ns}$ ). Final cubic box size values are $L=55.4,54.9,46.9$, and $36.7 \AA$ for the protonated in water, neutral and IL systems, and pair and single cation dendrimers, respectively. In all simulations, the periodic boundary conditions are implemented by using a neighbor atom list according to the link-cell algorithm and employing the smooth particle mesh Ewald method ${ }^{18}$ with a precision of $1 \times$ $10^{-6}$. The variation of the radius of gyration along the simulations is also presented as Supporting Information. We have verified that, in the final part of the trajectories, the dendrimer sizes show stable oscillations around their mean 
values. The total energy and temperature are similarly stable (see also Supporting Information). The final part used for analysis includes at least $7.5 \mathrm{~ns}$.

From the final trajectories we have analyzed different density profiles (for the dendrimer, water molecule, or anions). These profiles are defined as the radial density distribution ( $\rho$, in macroscopic units) and are obtained by analyzing the distance of the given atoms from each dendrimer center, $r$. Some examples showing the stability of these profile values along the final part of the trajectories are also given as Supporting Information. (Different ranges of oscillation are due to the obvious dependence of the sampling quality on the considered particle mobility and $r$.)

\section{RESULTS AND DISCUSSION}

a. Dendrimers in Water. In previous simulations for PAMAM-EDA with the Dreiding force field, the experimental dendrimer sizes could only be reproduced through an explicit description of hydrogen bonds. We have verified that the direct use of the PCFF for the dendrimer atom interactions results in smaller dendrimer sizes that show a substantial variation with $\mathrm{pH}$. Something similar was previously found with the first version of Dreiding. ${ }^{5}$ Consequently, we also have incorporated hydrogen bonds within the original PCFF. We have explored a simple modification based on the functional form and parameters considered in the hydrogen bond terms proposed for Dreiding. These parameters were calculated by performing ab initio quantum chemistry calculations. ${ }^{8}$ The hydrogen bond terms depend on the distance between donor and acceptor atoms, $R$, and also on the angle formed by their respective bonds with the $\mathrm{H}$ atom, $\theta$. In our calculations, we use a $6: 9$ Lennard-Jones that closely reproduces the functional form described in ref 8 for the distance dependence. Introducing the hydrogen bond terms according to these specifications as

$$
E(R, \theta)=D_{0}\left[2\left(R / R_{0}\right)^{9}-3\left(R / R_{0}\right)^{6}\right] \cos ^{2}(\theta)
$$

we have obtained averaged sizes for the protonated and neutral dendrimer closer to those calculated without the modification, i.e., values significantly smaller than the experimental result. However, we have found that the experimental data are closely reproduced when the different values for the potential well parameter, $D_{0}$, are reduced to a third of the values employed in the Dreiding force field ${ }^{8}$ (it seems that PCFF is able to partially take care of the hydrogen bond effects in more efficient way than Dreiding so that it requires a smaller quantitative modification). The potential parameters used in our final simulations are given in Table 1 .

Table 2 contains a summary of the simulation and experimental root-mean quadratic radius of gyration (representing the dendrimer size) results for the neutral and protonated G4 dendrimer in water (with $\mathrm{Cl}^{-}$counterions). It can be observed that our data are similar to those yielded by the previous simulations using the modified Dreiding force field. ${ }^{8}$ As discussed above, better agreement with the experimental sizes is obtained in the cases where the force fields include an explicit description of hydrogen bonds. Nevertheless, our results are slightly smaller than the most recent SANS experimental data, ${ }^{6}$ showing a greater $\mathrm{pH}$ (or protonation) influence on the dendrimer size. It should be pointed out, however, that this difference mimics the trend shown by the most recent experimental data corresponding to other PAMAM
Table 1. Hydrogen Bond Terms Added to the PCFF, According to Eq 1

\begin{tabular}{lcc} 
donor $\cdots$ acceptor groups & $D_{0}(\mathrm{kcal} / \mathrm{mol})$ & $R_{0}(\AA)$ \\
$\left(\mathrm{NH}_{4}\right)^{+} \cdots \mathrm{Cl}^{-}$ & 2.53 & 3.27 \\
$\left(\mathrm{NR}_{3} \mathrm{H}\right)^{+} \cdots \mathrm{Cl}^{-}$ & 3.33 & 2.98 \\
amide $\cdots \mathrm{Cl}^{-}$ & 1.87 & 3.26 \\
$\left(\mathrm{NR}_{3} \mathrm{H}\right)^{+} \cdots$ water oxygen & 0.74 & 3.12 \\
$\left(\mathrm{NH}_{4}\right)^{+} \cdots$ water oxygen & 0.41 & 3.20 \\
amide $\cdots$ water oxygen & 0.46 & 3.17 \\
water $\cdots$ amide oxygen & 0.44 & 3.15 \\
$\left(\mathrm{NH}_{4}\right)^{+} \cdots$ amide oxygen & 2.85 & 2.64 \\
$\left(\mathrm{NR}_{3} \mathrm{H}\right)^{+} \cdots$ amide oxygen & 2.79 & 2.77 \\
amide $\cdots$ amide oxygen & 1.29 & 2.90 \\
$\left(\mathrm{NH}_{3}\right) \cdots$ water oxygen & 0.44 & 3.41 \\
$\left(\mathrm{NH}_{3}\right) \cdots$ amide oxygen & 0.42 & 3.41 \\
water $\cdots\left(\mathrm{NR}_{3}\right)$ & 0.42 & 3.15 \\
$\left(\mathrm{NH}_{3}\right) \cdots\left(\mathrm{NR}_{3}\right)$ & 0.06 & 3.90 \\
amide $\cdots\left(\mathrm{NR}_{3}\right)$ & 0.14 & 3.40 \\
water $\cdots\left(\mathrm{NH}_{3}\right)$ & 0.66 & 3.12 \\
$\left(\mathrm{NH}_{3}\right) \cdots\left(\mathrm{NH}_{3}\right)$ & 0.31 & 3.47 \\
amide $\cdots\left(\mathrm{NH}_{3}\right)$ & 0.81 & 3.15 \\
\hline
\end{tabular}

dendrimers with different generation numbers, $G=3, G=5-$ $8^{10}$

In Table 2, we also show some results obtained without explicit hydrogen bonds in the force fields. Our data were obtained with the same open initial conformations used in the explicit hydrogen bond calculations. The evolution of these data is also included as Supporting Information. The final values have been obtained by averaging over the final $4 \mathrm{~ns}$. An extensive initial increase can be observed in the case of the protonated dendrimer. We have verified that the final equilibrium values obtained from these trajectories are significantly smaller than those calculated with explicit hydrogen bonds, both for the protonated and neutral cases. Nevertheless, there is also agreement between the results obtained with the original Dreiding and PCFF results, i.e., when hydrogen bonds are not included.

We should remark that our work is not focused on reaching a conclusive agreement between simulation and experimental size data for the water solution systems, because this agreement probably still require very extensive numerical work and maybe also more experimental work. However, the present calculations for the water solution systems confirm that our molecular model for the PAMAM molecules is able to yield reasonable data, providing us with a useful reference to compare with similar results corresponding to the ionic liquid system.

From the simulation trajectories, we have analyzed different conformational properties that may help us to describe the space distribution of atoms for the dendrimer solutions in water. In Figure 2, we show the dendrimer density profiles. The neutral dendrimer shows a peak at low distances and greater density at intermediate distances. This density, however, becomes smaller in the periphery region exhibiting an earlier decrease to zero density. The deep minimum and greater dendrimer density at the outer region for the protonated dendrimer seems to support a $\mathrm{pH}$-driven transition from "dense core" to "dense shell" configuration, as also described in the previous simulations. ${ }^{8}$ These different configurations could be considered to be reminiscent of the two alternative simplified 
Table 2. Root Mean Squared Radius of Gyration $(\AA)$ of $G=4$ PAMAM-EDA Dendrimers in Water

\begin{tabular}{|c|c|c|c|c|c|}
\hline & SANS $^{6}$ & Dreiding $^{5}$ & modified Dreiding ${ }^{8}$ & PCFF & modified PCFF \\
\hline neutral & $21.4 \pm 0.7$ & $16.8 \pm 0.2$ & $20.9 \pm 0.2$ & $16.84 \pm 0.05$ & $20.57 \pm 0.04$ \\
\hline protonated & $21.6 \pm 0.4$ & $19.0 \pm 0.1$ & $21.8 \pm 0.2$ & $19.3 \pm 0.1$ & $21.92 \pm 0.04$ \\
\hline
\end{tabular}

${ }^{a}$ With explicit hydrogen bond terms from Table 1 .

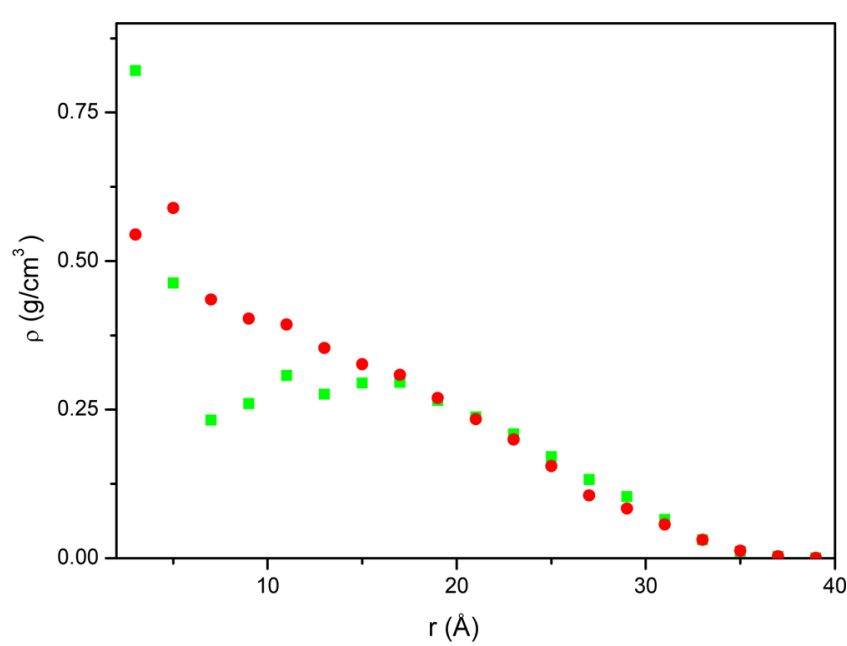

Figure 2. Dendrimer density profiles for the protonated and neutral dendrimers in water solution. Green squares: totally protonated dendrimers. Red circles: neutral dendrimers.

descriptions provided by the Boris-Rubinstein ${ }^{19}$ and de

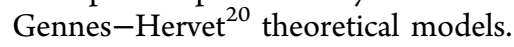

In Figure 3, we show the radial density distribution of water molecules at different distances from the dendrimer center. We

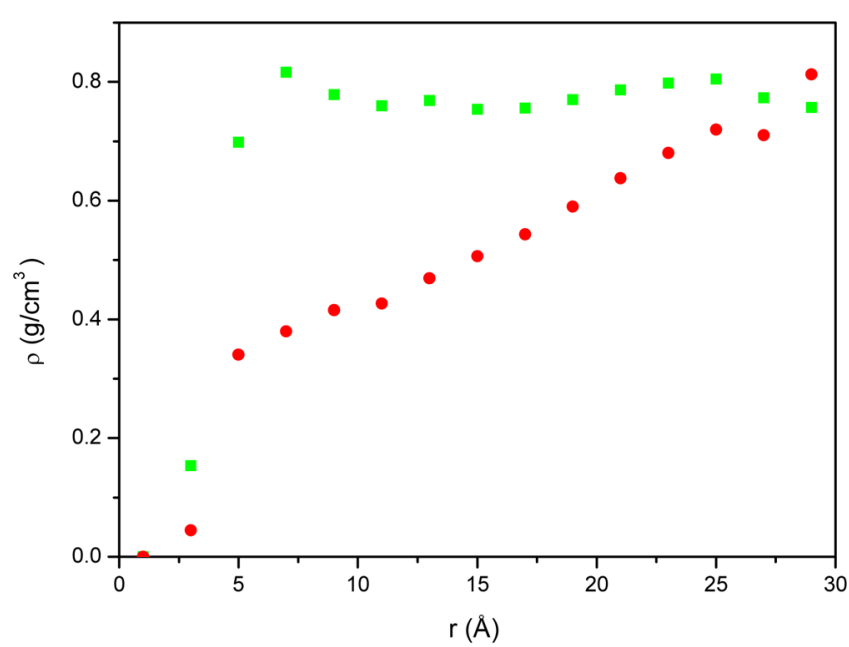

Figure 3. Water density profiles for the protonated and neutral dendrimers in water solution. Green squares: totally protonated dendrimers. Red circles: neutral dendrimers.

have applied the adequate geometrical correction in the regions closer to the box corners where only a part of the defined spherical shell is actually inside the sampled cube. For the region $L / 2<r<2^{-1 / 2} L$, which can be statistically significant in some cases, this correction can be simply computed from the volume corresponding to the six nonoverlapping spherical shell regions emerging out of the cube, $V_{\text {out }}=6 \pi h^{2}[3 r-h] / 3$, with $h$ $=r-L / 2$. A comparison of the differential volume of the spherical shell with that of the remaining part inside the box yields a correction factor of $2 r /(3 L-4 r)$.

The water density profiles corresponding to the neutral and protonated molecules exhibit more dramatic differences with respect to the results for the dendrimer profiles. They clearly show that water molecules can more easily approach the dendrimer center when the molecule is protonated. The protonated molecule also shows an early increase of the water density profile near the dendrimer center together with a flat aspect at intermediate distances and in the region outside the dendrimer. In the case of the neutral dendrimer, however, the water density is small in the core and at intermediate distances. It shows an abrupt increase in the dendrimer outer limits where both profiles tend to converge to the bulk density of water.

The water profile analysis, however, does not reveal how the water molecules are actually distributed inside and around the dendrimer. It can be claimed that some water molecules can be located close to the amine groups, interacting with them through hydrogen bonds. To verify this feature, we have analyzed distances between solvent molecules and the $\mathrm{N}$ atoms in the different amine groups, finding the closest amine to each water molecule. This allows us to discern the amine group where each water molecule seems to be located. Furthermore, we classify these amines according to the dendrimer generation shell they belong. The results are presented in Table 3 .

Table 3. Averaged Number of Water Molecules Closest to Different Amine Shells

\begin{tabular}{lcll} 
shell & amines & \multicolumn{1}{c}{ neutral } & protonated \\
0 (core) & 2 & $2.3 \pm 0.2$ & $27 \pm 1$ \\
1 & 4 & $17.6 \pm 1$ & $74 \pm 2$ \\
2 & 8 & $62.8 \pm 2$ & $156 \pm 2$ \\
3 & 16 & $220 \pm 5$ & $358 \pm 3$ \\
4 & 32 & $643 \pm 10$ & $822 \pm 5$ \\
5 (outer) & 64 & $3054 \pm 10$ & $2567 \pm 7$ \\
\hline
\end{tabular}

It can be verified that most of the water molecules remain closer to the outer dendrimer shell amines (obviously, most of the water is actually outside the dendrimer). However, a significant number of the water molecules are in the interior of the dendrimer. A higher number of water molecules are closer to the amine groups in the inner shells of protonated dendrimers. This is consistent with the water profile curves, which show that water molecules can more easily reach the dendrimer core of the protonated molecules. The neutral dendrimer exhibits a denser and uniform structure whereas the protonated dendrimer is more open and possibly segregated in dendrons and, consequently, water molecules can more easily permeate to locations closer to the protonated tertiary amine groups of the intermediate shells. According to a recent investigation combining scattering experiments and simulations, ${ }^{21}$ a significant number of $\mathrm{C}-\mathrm{H} \cdots \mathrm{O}$ bonds has been identified in the simulations (performed with the Amber software) for the protonated G5 dendrimer. These bonds have an important role in the system dynamics. 
Table 4. Root Mean Squared Radius of Gyration $(\AA)$ of $G=4$ PAMAM-EDA Dendrimers in the IL System

$\begin{array}{ccccc}\text { model } 1^{a} & \text { model } 2^{b} & \text { model } 3^{b} & {\text { model } 4^{c}}^{c} \text { single dendrimer }^{a} \\ 19.6 \pm 0.1 & 19.35 \pm 0.05 & 19.43 \pm 0.05 & 18.7 \pm 0.1\end{array}$

${ }^{a}$ No hydrogen bonds, anions initially adsorbed at amines. ${ }^{b}$ Hydrogen bonds (see text), anions initially adsorbed at amines. ${ }^{c}$ No hydrogen bonds, anions initially placed at random positions.

The shape of the dendrimer structures can be characterized by some numerical parameters, such as the averaged asphericity, $A$. The asphericity is obtained by calculating the eigenvalues, $\lambda_{i}$, of the tensor of mean quadratic components of the radius of gyration in a given configuration. More explicitly

$$
A=\frac{\left\langle\sum_{i>j}^{3}\left(\lambda_{i}-\lambda_{j}\right)^{2}\right\rangle}{\left\langle 2\left(\sum_{i=1}^{3} \lambda_{i}\right)^{2}\right\rangle}
$$

Values of $A$ close to zero indicate an approximately spherical shape. The protonated dendrimers in water shows a small value, $A \cong 0.02$, which indicates a nearly spherical form. This deviation is similar to our previous estimations ${ }^{22}$ for a single molecule represented by means of coarse-grained dendrimer models $^{23,24}$ in which the structure of repeat units was substituted by simple beads parametrized by using older SANS data of partially protonated PAMAM in water. However, the neutral dendrimer value, $A \cong 0.09$, represents a more noticeable deviation from the sphere. This asymmetry may be associated with the more compact structure of the neutral molecule.

b. IL Dendrimer System. Once our modified PCFF atomistic model for the PAMAM dendrimers has been shown to provide an acceptable description of the dendrimer-water system, we have applied it to the case of the protonated dendrimer acting as the cation component of an IL system. In this system, hydrogen bonds can be mainly of the type $\mathrm{NH} \cdots \mathrm{O}$, linking the different amine and amide $\mathrm{NH}$ groups in the dendrimer cation with the $\mathrm{O}$ atoms of the anion $\mathrm{S}=\mathrm{O}$ groups. $^{25}$ (The $\mathrm{H} \cdots \mathrm{N}$ and $\mathrm{H} \cdots \mathrm{F}$ interactions are relatively weak and they can be neglected. ${ }^{26}$ Quantum chemical calculations performed for the dimer formation of $\mathrm{Tf} 2 \mathrm{NH}^{25}$ yield a $\mathrm{H} \cdots \mathrm{O}$ equilibrium distance similar to the case of the $\mathrm{NH} \cdots \mathrm{O}$ hydrogen bond between amide groups included in Table 1 (the nitrogen atom in $\mathrm{Tf} 2 \mathrm{NH}$ is somewhat similar to a $\mathrm{N}$ atom in an amide group). On the basis of this result, we assume that the different hydrogen bond interactions with $\mathrm{O}$ atoms in the $\mathrm{S}=\mathrm{O}$ groups are similar to those corresponding to the amide groups in the dendrimer-water system. To study the influence of these hydrogen bond interactions on the IL system, we have also considered three alternative models. Model 1 does not consider hydrogen bond interactions, model 2 considers $\mathrm{O}$ atoms in amide and in $\mathrm{S}=\mathrm{O}$ groups to be totally equivalent, and model 3 introduces a factor of 2 in the interactions for $\mathrm{O}$ atoms in $\mathrm{S}=\mathrm{O}$ with respect to those in amide groups. (Model 3 is inspired by the high bond formation energies found in the quantum mechanics calculations for $\mathrm{Tf} 2 \mathrm{NH}$ dimers. $)^{25}$

In Table 4, we show the results obtained for the dendrimer size for these three models. Differences between the models are small. In the three cases, the values are smaller than those obtained for both neutral and protonated dendrimers in water when hydrogen bonds were included in the Dreiding force field or PCFF, even though in the IL system the dendrimer cation contains a certain number of inner localized relatively bulky anions. We also include in Table 4 our simulation results obtained with an initial configuration where anions are placed at random locations without explicit hydrogen bonds (model 4). It can be observed that, in this system, the mean size of the molecules is only slightly smaller. A further reduction in size is observed for the system with a single dendrimer cation. It should be noted, however, that the smaller box size of this system can result in significant self-interactions due to use of periodic boundary conditions.

In Figure 4 we present the single (or individual) dendrimer density profiles obtained with the four models. The profiles

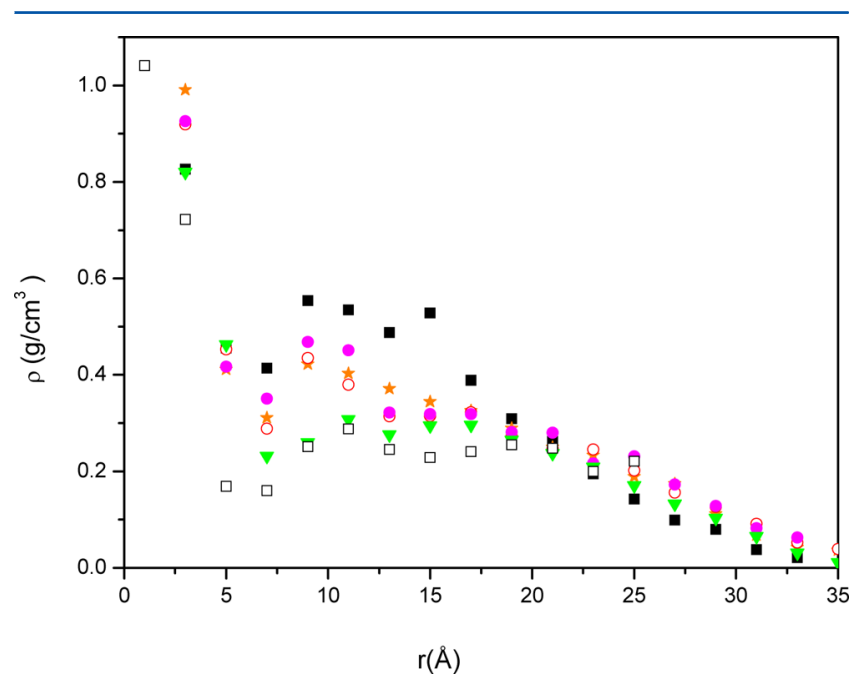

Figure 4. Dendrimer density profiles obtained with three different models (different strengths of the explicit hydrogen bond interactions) in our IL system with $\mathrm{Tf} 2 \mathrm{~N}^{-}$anions close to the amines: red open circles, model 1 (no hydrogen bonds explicitly included); orange stars, model 2; magenta solid circles, model 3; solid black squares, model 4 (no hydrogen bonds, anions initially placed at random); open black squares, single dendrimer IL system. The density profile corresponding to a protonated dendrimer in water (considering hydrogen bonds) is also included for comparison (green triangles).

obtained for models 1-3 are very similar, which seems to confirm the small effect of including explicit hydrogen bond interactions when the IL systems are described. Model 4, however, shows a more prominent peak at intermediate distances, associated to a broader and denser core. The results obtained for the protonated molecule in water and the single cation IL dendrimer are also included for comparison. The latter data show a minimum at intermediate distances for which all the two cation IL systems exhibit peaks. This suggests a structural difference between the protonated dendrimer in water and the IL cation due to the effect of interpenetration between the dendrimer molecules.

Figure 5 contains a summary of different profile curves corresponding to a given reference dendrimer, all of them as a function of the dendrimer center. Specifically, it shows the density of the dendrimer discussed above, the "reciprocal" density corresponding to atoms of the other dendrimer cation, the density of anions, and the total density of the system, The results correspond to model 1 where hydrogen bonds are not 


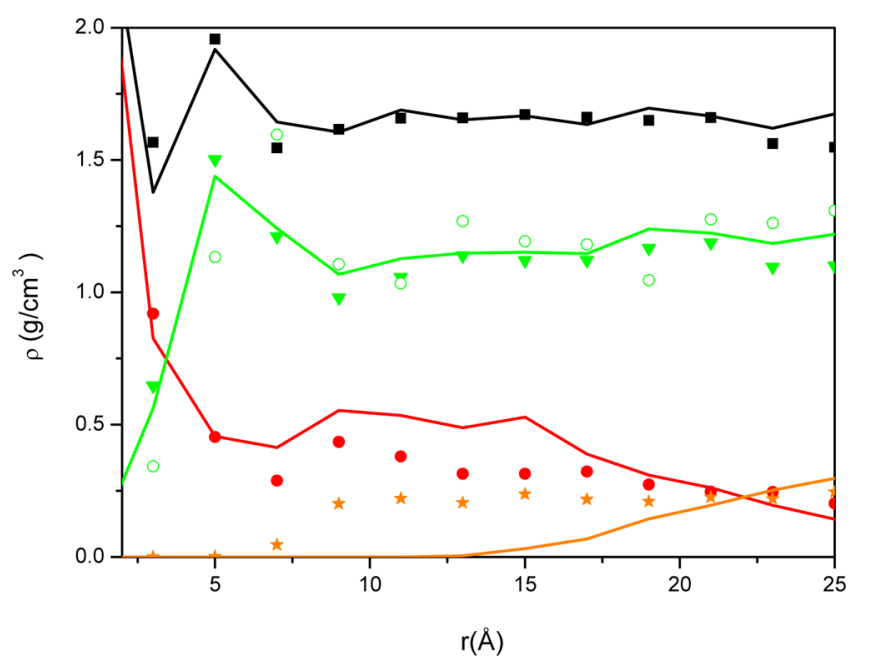

Figure 5. Different profile densities in the IL system composed of two dendrimer cations and $252 \mathrm{Tf}_{2} \mathrm{~N}^{-}$anions, model 1 (see text): red circles, internal dendrimer density; green triangles, anion density; orange stars, external dendrimer density; black squares, total density. Accompanying solid lines, same color codes: model 4 (anions initially placed at random). The anion density corresponding to the single dendrimer IL is also included (open green circles).

explicitly considered; we have not found any significant differences when hydrogen bonds are explicitly included (models 2 and 3 ). We also include the data corresponding to model 4 with a different starting configuration (random initial disposition of anions). We notice the obvious asymptotic approach to the bulk density of the anion and total density together with the expected decay in the density of the local dendrimer. More interesting, we can observe a peak for the anions density located close to the dendrimer core. In spite of this local feature, global density does not fluctuate much, which indicates a good steric accommodation of the bulky anions within the dendrimer cations. A similar anion profile is also observed for the single dendrimer IL system.

The "reciprocal" dendrimer profile does not show a noticeable peak reflecting a higher density at the second dendrimer center, probably because this is a local feature whose effect is diluted when the total system volume is considered. There is a surprisingly high degree of overlap between the cations, with a non-negligible density of the other dendrimer at less than $10 \AA$ from each dendrimer center for model 1 . For model 4 , the external dendrimer is absent at short distances from the center, apparently being replaced by internal atoms. However, we find similar densities for models 1 and 4 at distances from the dendrimer center equivalent to its radius of gyration (roughly a half of the local dendrimer mass is located at greater distances).
It should be considered that model 1 shows an important degree of initial dendrimer overlap because initially all the anions are placed close to the amine groups. However, initial overlap is small in model 4 , where most anions are initially placed between the two dendrimer molecules. We have verified that the distance between centers of masses is significantly greater than the distance between the dendrimer centers for model 1; this reflects an anisotropic displacement of the center of masses in the orientations opposite to the overlap. This overlap persists along the whole trajectory. However, both types of distance values are similar (within $2 \AA$ ) for model 4, where overlapping takes place during the first simulation steps. (The onset of overlapping for model 4 is included as Supporting Information.)

Therefore, model 4 is more useful to show that dendrimers show a noticeable degree of overlap at equilibrium in the dendrimer intermediate shells with an isotropic dendrimer internal distribution. A similar conclusion on symmetry is obtained by studying the dendrimer asphericities. For model 1 , $A \cong 0.07$, reflecting a distorted shape due to the asymmetric accommodation of the second dendrimer, whereas for model 4 $A \cong 0.005$; i.e., the dendrimers are practically spherical. For a real many-dendrimer system, we may expect that the dendrimer cations in IL show some type of ordering due to overlap, as that present in molecules with a central core and a flexible corona (i.e., in the case of highly armed star polymer melts ${ }^{27}$ ).

In our analysis of results, we have also investigated the location of anions, by identifying the amine group that is closest to the central $\mathrm{N}$ atom of each one of the $\mathrm{Tf} 2 \mathrm{~N}^{-}$structures for models 1 and 4 (again, we have found no significant differences between models $1-3$ ). The results are presented in Table 5, were we also include, for the sake of comparison, the location of the $\mathrm{Cl}^{-}$counterions around and within the protonated dendrimer in water. These results correspond to the average number of times that we find the closest amine group at the indicated internal generation shell. As a reference, we include the number of amines present in each one of these shells. One should keep in mind that each anion was initially positioned close to an amine group.

We can observe that both $\mathrm{Tf} 2 \mathrm{~N}^{-}$and $\mathrm{Cl}^{-}$anions tend to partially migrate to the outer part of the dendrimer. We find more than 90 anions closest to these outer primary amines in model 1 , whereas the number of amines in the outer shell is 64 . This value is significantly high, even if we admit that half of the 32 anions close to the shell 4 amines could also be accounted as closer to the outer shell 5 . It should be remarked that a similar amount of inner anions is found for model 4, where the anions were initially disposed at random. There are slight differences between the number of anions associated with the last shells and outside the dendrimer for models 1 and 4. However, taking into account the number of anions associated to the outer shell

Table 5. Averaged Number of Anions Closest to Different Amines

$\begin{array}{lcccc}\text { shell } & \text { amines } & \mathrm{Cl}^{-} \text {in water } & \text { Tf2N }^{-} \text {in IL, model } 1^{a} & \text { Tf2N }^{-} \text {in IL, model } 4^{b} \\ 0 & 2 & 0.5 \pm 0.5 & 1.69 \pm 0.05 & 1.62 \pm 0.02 \\ 1 & 4 & 1.5 \pm 0.1 & 1.7 \pm 0.1 & 1.53 \pm 0.04 \\ 2 & 8 & 2.9 \pm 0.2 & 5.4 \pm 0.1 & 4.7 \pm 0.1 \\ 3 & 16 & 7.5 \pm 0.2 & 8.8 \pm 0.1 & 9.5 \pm 0.1 \\ 4 & 32 & 27.7 \pm 0.5 & 16.0 \pm 0.2 & 10.4 \pm 0.1 \\ 5 \text { (outer) } & 64 & 85.9 \pm 0.5 & 92.4 \pm 0.2 & 98.7 \pm 0.5\end{array}$

${ }^{a}$ No hydrogen bonds, anions initially adsorbed at amines. ${ }^{b}$ No hydrogen bonds, anions initially placed at random positions. 
in the initial configurations in these models (78 and 93, respectively), it is clear that a certain number of anions prefer to be located in the dendrimer periphery instead of staying adsorbed close to the amine groups. (The single dendrimer system also shows a clear migration to the outer shells 4 and 5.) The excess number of anions in the outer part of dendrimers in the IL system favors the interpenetration between different dendrimer cations. Moreover, the nonhomogeneous distribution of charges may have an important influence in the ionic conductivity of the system.

The inner shell 4 contains more $\mathrm{Cl}^{-}$ions in the water system than $\mathrm{Tf} 2 \mathrm{~N}^{-}$anions in the $\mathrm{IL}$ system. However, the $\mathrm{Cl}^{-}$anions have less preference to remain within more profound intermediate inner shells. These differences may be related to the greater facility of the smaller $\mathrm{Cl}^{-}$anions to move. Note that this conclusion is obtained for two different initial distributions of anions, models 1 and 4 (and also in the case of the single dendrimer system). Therefore, the $\mathrm{Tf} 2 \mathrm{~N}^{-}$anions show a greater degree of location close to the deeper ternary amines than simple anions. This feature may have a role in the noticeable blue photoluminescence observed for the IL system. $^{4}$ Fluorescence emission of PAMAM solutions is known to increase at lower $\mathrm{pH}$ (higher ternary amine protonation) and it may be associated with the amine oxidation. $^{28}$

\section{CONCLUSIONS}

Our simulations use a modified version of the PCFF considering the option of an explicit term for hydrogen bonds for the PAMAM dendrimer. To validate this model, we have performed simulations for protonated and neutral dendrimers in water. These simulations provide results remarkably close to recent simulations that also include hydrogen bonds within the Dreiding force field, showing an adequate reproduction of the SANS data for the $G=4$ protonated and neutral dendrimers. The difference between the dendrimer sizes obtained for these two types of systems is greater than that found experimentally. However, it is consistent with the SANS results corresponding to other generation numbers. The internal structure of the protonated molecules seems to be more open, spherically symmetric, and maybe separated in dendrons. Therefore, water molecules have an easier approach to the core.

The main goal of our work has been to study the structure of the IL dendrimers. Our simulations show dendrimer size smaller than experimentally found in the water solutions, even when explicit hydrogen bonds are included in the model. Also adopting the water systems as references, the dendrimer density profile shows some differences. The two dendrimer cations included in our system exhibit a noticeable degree of overlap that can be favored by the presence of an abundant number of anion molecules in the peripherical regions of the dendrimer molecules. Considering the intermediate inner generation shells closer to the dendrimer core, the $\mathrm{Tf} 2 \mathrm{~N}^{-}$anions show a greater degree of location close to the ternary amines than the simple anions such as $\mathrm{Cl}^{-}$in the water system.

\section{ASSOCIATED CONTENT}

\section{S Supporting Information}

Figures S1-S14 of variation of different properties with time along the simulation runs: global system density, dendrimer size, system temperature, system total energy, cation dendrimer density profile, water density profile, anion density profile, density of a second dendrimer, distance between the dendrimer centers and center of masses. This material is available free of charge via the Internet at http://pubs.acs.org.

\section{AUTHOR INFORMATION}

\section{Corresponding Author}

*J. Freire: e-mail, jfreire@invi.uned.es.

\section{Notes}

The authors declare no competing financial interest.

\section{ACKNOWLEDGMENTS}

This work has been partially supported by Projects CTQ201016414 from "Ministerio de Economía y Competitividad, DGICT" and P2009/ESP-1691 of the CAM (Spain) and "UNED Postdoctoral Grants" (for AA and CM).

\section{REFERENCES}

(1) Fréchet, J. M. J.; Tomalia, D. A. Dendrimers and Other Dendritic Polymers; Wiley: Chichester, England, 2001.

(2) Freire, J. J. Soft Matter 2008, 4, 2139.

(3) Freemantle, M. An Introduction to Ionic Liquids; The Royal Society of Chemistry: Cambridge, U.K., 2010.

(4) Huang, J.-F.; Luo, H.; Liang, C.; Sun, I.-W.; Baker, G. A.; Dai, S. J. Am. Chem. Soc. 2005, 127, 12784.

(5) Maiti, P. K.; Çă̆in, T.; Lin, S.-T.; Goddard, W. A., III. Macromolecules 2005, 38, 979.

(6) Chen, W.-R.; Porcar, L.; Liu, Y.; Butler, P. D.; Magid, L. J. Macromolecules 2007, 40, 5887.

(7) Nisato, G.; Ivkov, R.; Amis, E. J. Macromolecules 2000, 33, 4172.

(8) Liu, Y.; Bryantsev, V. S.; Diallo, M. S.; Goddard, W. A., III. J. Am. Chem. Soc. 2009, 131, 2798.

(9) Mayo, S. L.; Olafson, B. D.; Goddard, W. A., III. J. Phys. Chem. 1990, 94, 8897.

(10) Liu, Y.; Chen, C.-Y.; Chen, H.-L.; Hong, K.; Shew, C.-Y.; Li, X.; Liu, L.; Melnichenko, Y. B.; Smith, G. S.; Herwig, K. W.; Porcar, L.; Chen, W.-R. J. Phys. Chem. Lett. 2010, 1, 2020.

(11) Sun, H.; Mumby, S. J.; Maple, J. R.; Hagler, A. T. J. Am. Chem. Soc. 1994, 116, 2978

(12) Jorgensen, W. L.; J. Chandrasekhar, J.; Madura, J. D.; Impey, R. W.; Klein, M. L. J. Chem. Phys. 1983, 79, 926.

(13) Borodin, O.; Smith, G. J. Phys. Chem. B 2006, 110, 11481.

(14) Accelrys Software Inc., San Diego, CA.

(15) Rappe, A. K.; Goddard, W. A., III. J. Chem. Phys. 1991, 95, 3358.

(16) Smith, W.; Yong, C. W.; Rodger, P. M. Mol. Simul. 2002, 28, 385 .

(17) Ahmadi, A.; McBride, C.; Freire, J. J.; Kajetanowicz, A.; Czaban, J.; Grela, C. J. Phys. Chem. A 2011, 115, 12017.

(18) Essmann, U.; Perera, L.; Berkowitz, M. L.; Darden, T.; Lee, H.; Pedersen, L. G. J. Chem. Phys. 1995, 103, 8577.

(19) Boris, D.; Rubinstein, M. Macromolecules 1996, 29, 7251.

(20) de Gennes, P.-G.; Hervert, H. J. Phys. Lett. 1983, 44, L351.

(21) Wu, B.; Liu, Y.; Li, X.; Mamontov, E.; Kolesnikov, A.; Diallo, S. A.; Do, C.; Porcar, L.; Hong, K.; Smith, S. C.; Liu, L.; Smith, G. S.; Egami, T.; Chen, W.-R. J. Am. Chem. Soc. 2013, 135, 5111.

(22) Freire, J. J.; Rubio, A. M. Polymer 2008, 49, 2762.

(23) Freire, J. J.; Rodríguez, E.; Rubio, A. M. J. Chem. Phys. 2005, 123, 1549901.

(24) Del Río Echenique, G.; Rodríguez Schmidt, R.; Freire, J. J.; Hernández Cifre, J. G.; García de la Torre, J. J. Am. Chem. Soc. 2009, $131,8548$.

(25) Chipanina, N. N.; Sterkhova, I. V.; Aksamentova, T. N.; Sherstyannikova, L. V.; Kukhareva, V. A.; Shainyan, B. A. Russ. J. Gen. Chem. 2008, 78, 2363.

(26) Qiao, B.; Krekeler, C.; Berger, R.; Site, L. D.; Holm, C. J. Phys. Chem. B 2008, 112, 1743.

(27) Di Cecca, A.; Freire, J. J. Macromolecules 2002, 35, 2851. 
(28) Wang, D. J.; Imae, T.; Miki, M. J. Colloid Interface Sci. 2007, 312, 